\title{
Representation of the pulsed output from a mode-locked laser using quantum field theory and an application in multiphoton ionisation
}

\author{
D L Andrews \\ Departments of Mathematics and Chemistry, University College London, Gower Street, \\ London WC1E 6BT, England
}

Received 6 February 1978, in final form 30 March 1978

\begin{abstract}
The pulsed output obtainable from a mode-locked laser has usually been described using classical radiation theory. In this paper a quantised-field representation is developed and utilised in a discussion of multiphoton ionisation. The assumptions made in deriving the results are critically examined, and a modification of the theory to deal with other kinds of pulsed radiation is indicated.
\end{abstract}

\section{Introduction}

Mode-locked lasers are being used for an increasingly wide range of applications. Their most important feature is the emission of light in pulses of extremely short duration, typically in the picosecond range (Greenhow and Schmidt 1974, Shapiro 1977). Since the instantaneous intensity of these pulses can reach very high peak values, mode-locked lasers are generally more effective than continuous-wave lasers in the study of non-linear processes such as multiphoton ionisation, and they have accordingly been used in much of the important research in this field (Lompre et al 1976, 1977). Although few theoretical studies have been specifically addressed to interactions on the picosecond time scale, interesting new effects have recently been predicted for resonance ionisation induced by ultrashort pulses (Andrews 1977).

For a complete analysis of processes commonly studied with picosecond pulses, it is clearly necessary to model the rapid temporal variation in the amplitude of the electric field. For this purpose classical radiation theory has usually been employed, as the difficulty of representing pulses in quantum field theory has long been appreciated (see, for example, Crance and Feneuille 1977). In this paper, however, a quantised-field representation of mode-locked pulses is developed, and its application is demonstrated in a discussion of multiphoton ionisation. A modification of the theory to deal with the single pulses of radiation from a giant-pulse laser is also indicated. Finally, the assumptions made in the course of deriving the results are critically examined, and a cautionary remark is made concerning the use of subpicosecond pulses in ionisation studies.

\section{Representation of mode-locked pulses using quantum field theory}

The permitted wavelengths $\lambda$ of the stationary axial modes in a laser cavity of optical path length $L$ are governed by the elementary relation $m(\lambda / 2)=L$, where $m$ is an 
integer. The corresponding circular frequencies are given by $\omega=m(\pi c / L)$, and the frequency interval between adjacent modes is $\Omega=\pi c / L$. For simplicity, we consider the idealised case where all modes have the same polarisation, as well as collinear propagation vectors, so that each mode is uniquely labelled by its frequency. The laser output at any particular frequency is determined by the gain, which we assume exceeds the threshold over a domain centred on some frequency $\omega_{0}$, encompassing a further $M$ modes on either side of $\omega_{0}$. The laser output thus has frequency components

$$
\omega_{l}=\omega_{0}+l \Omega \quad-M \leqslant l \leqslant M
$$

and the spectral distribution is generally symmetric about a maximum at $\omega_{0}$. In a mode-locked laser there is a definite phase relationship between the axial modes which normally results in a pulsing of the output: it is the temporal representation of these pulses with which we are concerned in this section. For this purpose we adopt the simple model of a one-atom photon detector (Nussenzveig 1973), in which the rate of single-photon absorption is used as a measurement of the irradiance (power per unit area) of the laser beam.

In order to describe pulses of laser radiation in a quantised-field framework, it is desirable to employ states of the radiation field which closely approximate to the classical-wave representation of the laser output: coherent states (Glauber 1963) are well suited to this purpose, and are therefore used in the theory which follows. Unlike the commonly used number states $|n(\omega)\rangle$, for which the number of photons $n$ in a mode of frequency $\omega$ is specified exactly, the coherent states $|\alpha(\omega)\rangle$ do not have sharp values for the mode occupation numbers. Coherent states are, instead, characterised by the complex quantities $\alpha(\omega)$, the mean number of photons $\bar{n}(\omega)$ being given by

$$
\bar{n}(\omega)=|\alpha(\omega)|^{2}
$$

The state vector for the laser radiation field may be written as a product of coherent states for each of the frequency components, as follows;

$$
|r\rangle=\prod_{l=-M}^{M}\left|\alpha\left(\omega_{l}\right)\right\rangle
$$

with $\omega_{l}$ given by equation (1).

Now in the classical limit, the coherent state $\left|\alpha\left(\omega_{l}\right)\right\rangle$ corresponds to a wave with a phase angle equal to the phase of $\alpha\left(\omega_{l}\right)$ (Loudon 1973). In the particular case where this phase depends linearly on the mode index $l$, the electric field is said to be perfectly mode locked (Garmire and Yariv 1967, Grütter et al 1969): we restrict attention to this case in the following. Thus if we write

$$
\alpha\left(\omega_{l}\right)=\left|\alpha\left(\omega_{l}\right)\right| \exp \left[-\mathrm{i} \phi\left(\omega_{l}\right)\right]
$$

then the condition for perfect mode locking may be represented in a general way by the equation

$$
\phi\left(\omega_{l}\right)=\omega_{l} t_{m}+\phi_{0}
$$

where $t_{m}$ and $\phi_{0}$ are constants. 
The electric field operator $e^{\perp}$ may be expressed in terms of a mode expansion taking the form

$$
e^{\perp}=\sum_{\kappa, \lambda}\left(\frac{2 \pi \hbar c \kappa}{V}\right)^{1 / 2} \mathrm{i}\left[e^{(\lambda)}(\boldsymbol{\kappa}) a^{(\lambda)}(\boldsymbol{\kappa})-\bar{e}^{(\lambda)}(\boldsymbol{\kappa}) a^{(\lambda) \dagger}(\boldsymbol{\kappa})\right]
$$

at the spatial coordinate origin. Here $a^{(\lambda)}(\boldsymbol{\kappa})$ and $a^{(\lambda) \dagger}(\boldsymbol{\kappa})$ are the annihilation and creation operators, respectively, for a photon of wavevector $\boldsymbol{\kappa}$ and polarisation $e^{(\lambda)}$; $V$ is the volume of the quantisation box. Equation (6) may be written as $e^{\perp}=e^{(+)}+e^{(-)}$, where $e^{(+)}$contains the annihilation operators and $e^{(-)}$the creation operators. In the rotating-wave approximation only the former is involved in absorption, and the interaction operator is therefore

$$
H_{\text {int }}=-\mu \cdot e^{(+)} \text {. }
$$

We now calculate the single-photon absorption rate. In order to obtain the absorption at time $t$, we first calculate the probability amplitude for the transition $|k, f\rangle \leftarrow|i, r\rangle$, where $|a, b\rangle$ represents the product of an atomic-state vector $|a\rangle$ and a radiation-state vector $|b\rangle$; later, we sum over an appropriate set of final states $|k\rangle$ and $|f\rangle$ for the atom and the field. The probability amplitude is given by

$$
c\left(f, k: t \mid i, r: t_{0}\right)=\left\langle f, k\left|U\left(t, t_{0}\right)\right| i, r\right\rangle
$$

with the time-evolution operator $U\left(t, t_{0}\right)$ expressible as the series

$U\left(t, t_{0}\right)=1+\sum_{n=1}^{\infty}\left(\frac{1}{\mathrm{i} \hbar}\right)^{n} \int_{t_{0}}^{t} \mathrm{~d} t_{1} \int_{t_{0}}^{t_{1}} \mathrm{~d} t_{2} \ldots \int_{t_{0}}^{t_{n}-1} \mathrm{~d} t_{n} \tilde{H}_{\mathrm{int}}\left(t_{1}\right) \tilde{H}_{\mathrm{int}}\left(t_{2}\right) \ldots \tilde{H}_{\mathrm{int}}\left(t_{n}\right)$.

Here, $\tilde{H}_{\text {int }}(t)$ is given by

$$
\tilde{H}_{\mathrm{int}}(t)=\exp \left[\mathrm{i} H_{0}\left(t-t_{0}\right) / \hbar\right] H_{\mathrm{int}} \exp \left[-\mathrm{i} H_{0}\left(t-t_{0}\right) / \hbar\right]
$$

where

$$
H_{0}=H_{\mathrm{atom}}+H_{\mathrm{rad}}
$$

and $t_{0}$ is the time origin for absorption measurements. The dominant contribution to $(8)$ for single-photon absorption is

$c^{(1)}\left(f, k: t \mid i, r: t_{0}\right)$

$$
\begin{aligned}
= & \frac{\mathrm{i}}{\hbar} \int_{t_{0}}^{t} \mathrm{~d} t_{1}\left\langle k\left|\exp \left[\mathrm{i} H_{\mathrm{atom}}\left(t_{1}-t_{0}\right) / \hbar\right] \mu_{\lambda} \exp \left[-\mathrm{i} H_{\mathrm{atom}}\left(t_{1}-t_{0}\right) / \hbar\right]\right| i\right\rangle \\
& \times\left\langle f\left|\exp \left[\mathrm{i} H_{\mathrm{rad}}\left(t_{1}-t_{0}\right) / \hbar\right] e_{\lambda}^{(+)} \exp \left[-\mathrm{i} H_{\mathrm{rad}}\left(t_{1}-t_{0}\right) / \hbar\right]\right| r\right\rangle
\end{aligned}
$$

using the implied summation convention for the components of $e^{(+)}$and the atomic dipole-moment operator $\mu$. The atomic part of (12) can immediately be re-expressed through the equation

$$
\left\langle k\left|\exp \left[\mathrm{i} H_{\mathrm{atom}}\left(t_{1}-t_{0}\right) / \hbar\right] \mu_{\lambda} \exp \left[-\mathrm{i} H_{\mathrm{atom}}\left(t_{1}-t_{0}\right) / \hbar\right]\right| i\right\rangle=\mu_{\lambda}^{k i} \exp \left[\mathrm{i} \omega_{k i}\left(t_{1}-t_{0}\right)\right]
$$

where $\omega_{k i} \equiv\left(E_{k}-E_{i}\right) / \hbar$. 
From the electric field expansion (6), and equations (1) and (5), it can be shown that the radiation part of (12) can be written as

$$
\begin{aligned}
& \left\langle f\left|\exp \left[\mathrm{i} H_{\mathrm{rad}}\left(t_{1}-t_{0}\right) / \hbar\right] e_{\lambda}^{(+)} \exp \left[-\mathrm{i} H_{\mathrm{rad}}\left(t_{1}-t_{0}\right) / \hbar\right]\right| r\right\rangle \\
& =\left(\frac{2 \pi \hbar}{V}\right)^{1 / 2}\langle f \mid r\rangle \mathrm{i} e_{\lambda} J\left(t_{1}\right) \exp \left\{-\mathrm{i}\left[\omega_{0}\left(t_{1}+t_{m}-t_{0}\right)+\phi_{0}\right]\right\} .
\end{aligned}
$$

Here $e$ is the polarisation vector of the laser radiation, and $J(t)$ is a temporal envelope function, defined by

$$
J(t)=\sum_{l=-M}^{M} a_{l} \exp \left[-\mathrm{i} l \Omega\left(t+t_{m}-t_{0}\right)\right]
$$

with coefficients $a_{l}$ given by

$$
a_{l}=\left|\alpha\left(\omega_{l}\right)\right| \omega_{l}^{1 / 2} \text {. }
$$

Provided the distribution of energy between the $(2 M+1)$ modes in the laser output is symmetric about the circular frequency $\omega_{0}, J(t)$ is a real function. With equations (13) and (14), the probability amplitude (12) may be expressed in terms of $J(t)$ as follows:

$$
\begin{aligned}
& c^{(1)}\left(f, k: t \mid i, r: t_{0}\right) \\
&=-\left(\frac{2 \pi}{\hbar V}\right)^{1 / 2}\left(\boldsymbol{\mu}^{k i} \cdot e\right)\langle f \mid r\rangle \exp \left[-\mathrm{i}\left(\omega_{k i}-\omega_{0}\right) t_{0}\right] \\
& \times \exp \left[-\mathrm{i}\left(\omega_{0} t_{m}+\phi_{0}\right)\right] \int_{t_{0}}^{t} J\left(t_{1}\right) \exp \left[\mathrm{i}\left(\omega_{k i}-\omega_{0}\right) t_{1}\right] \mathrm{d} t_{1} .
\end{aligned}
$$

A detailed discussion of the envelope function is given in the appendix, but certain features should be mentioned at this stage. First, we note that $J(t)$ is essentially a truncated Fourier series, which can be written as

$$
J(t)=\sum_{l=-\infty}^{\infty} a_{l} \exp \left[-\mathrm{i} l \Omega\left(t+t_{m}-t_{0}\right)\right]
$$

with

$$
a_{l} \simeq 0 \quad|l|>M .
$$

The expansion interval for this series is $\left(t_{0}-t_{m}-T / 2, t_{0}-t_{m}+T / 2\right)$, where $T$ is the period defined by $T=2 \pi / \Omega=2 L / c$, which is the round-trip time for the laser cavity. In general, $J(t)$ exhibits a strong peak at the centre of the expansion interval, so that successive peaks are found at times $t=t_{0}-t_{m}+n T$. Also $J(t) \simeq 0$ in the troughs between these peaks; thus $J(t)$ properly represents a train of light pulses.

A second remark concerns the time origin $t_{0}$ for the absorption measurements. Since it is desirable for this to coincide with the onset of one of the pulses, a value for $t_{m}$ should be chosen which ensures that $t_{0}$ lies between two adjacent peaks in the pulse train. To this end we may set

$$
t_{m}=-T / 2
$$

giving pulses at odd multiples of $T / 2$ after $t_{0}$, and

$$
J\left(t_{0}\right) \simeq 0 \text {. }
$$


For generality, however, we do not make use of (20) in the calculations, though the condition (21) is necessary for a smooth switching-on of the photodetection process.

The photodetection probability may now be calculated using the formula

$$
P(t)=\sum_{f} \int\left|c\left(f, k: t \mid i, r: t_{0}\right)\right|^{2} \rho_{k} \mathrm{~d} E_{k}
$$

where $\rho_{k}$ is the density of states around the continuum energy level $E_{k}$. The summation over final states of the radiation field in (22) is necessary since no measurement is made on the field after the absorption. The summation is therefore taken over a complete set of states for each mode of the field, and the completeness relation is directly applicable. Hence equations (17) and (22) give

$$
P(t)=\frac{2 \pi}{V} \int_{t_{0}}^{t} \mathrm{~d} t_{1} \int_{t_{0}}^{t} \mathrm{~d} t_{1}^{\prime} J\left(t_{1}\right) J\left(t_{1}^{\prime}\right) \int \mathrm{d} \omega_{k}\left|\boldsymbol{\mu}^{k i} \cdot e\right|^{2} \rho_{k} \exp \left[\mathrm{i}\left(\omega_{k i}-\omega_{0}\right)\left(t_{1}-t_{1}^{\prime}\right)\right]
$$

and the usual Fermi-rule approximations can be made, leading to the result

$$
P(t)=\frac{4 \pi^{2}}{V}\left|\mu^{k i} \cdot e\right|^{2} \rho_{k} \int_{t_{0}}^{t} J^{2}\left(t_{1}\right) \mathrm{d} t_{1} .
$$

The instantaneous rate of absorption $R(t)$ is now given by the time derivative of (23), and may be written as

$$
R(t)=\frac{4 \pi^{2}}{\hbar c}\left|\mu^{k i} \cdot e\right|^{2} \rho_{k} I(t)
$$

where $I(t)$ is the irradiance defined by

$$
I(t)=\frac{\hbar c}{V} J^{2}(t)
$$

Taking (25) as the quantum electrodynamical definition of the time-dependent irradiance, we now proceed with a discussion of multiphoton ionisation, starting with the simplest two-photon case.

\section{Two-photon ionisation}

In this section we consider the case where the combined energy of any two photons in the beam is sufficient to match or exceed the ionisation potential, so that the absorption of two photons is the dominant mechanism for ionisation. Our conditions are thus represented by the inequalities

$$
\hbar \omega_{M}<W \leqslant 2 \hbar \omega_{-M}
$$

where $W$ is the first ionisation potential of the atom. The two inequalities in (26) together imply $\Delta \omega<\frac{1}{3} \omega_{0}$, where $\Delta \omega$ is the bandwidth $M \Omega$; this is a physically reasonable requirement.

For two-photon ionisation the probability amplitude $c^{(2)}\left(f, k: t \mid i, r: t_{0}\right)$ is given by equation (8) with the $n=2$ term in the expansion of the time-evolution operator, equation (9). If $|I\rangle$ represents an atomic intermediate state and $|n\rangle$ an intermediate 
state of the radiation field, then we may write

$$
\begin{aligned}
c^{(2)}(f, k: t \mid i, r: & \left.t_{0}\right) \\
= & \frac{\mathrm{i}}{\hbar} \sum_{n} \sum_{I} \int_{t_{0}}^{t} \mathrm{~d} t_{1}\langle k| \exp \left[\mathrm{i} H_{\text {atom }}\left(t_{1}-t_{0}\right) / \hbar\right] \mu_{\lambda} \\
& \times \exp \left[-\mathrm{i} H_{\mathrm{atom}}\left(t_{1}-t_{0}\right) / \hbar\right]|I\rangle\langle f| \exp \left[\mathrm{i} H_{\mathrm{rad}}\left(t_{1}-t_{0}\right) / \hbar\right] e_{\lambda}^{(+)} \\
& \times \exp \left[-\mathrm{i} H_{\mathrm{rad}}\left(t_{1}-t_{0}\right) / h\right]|n\rangle c^{(1)}\left(n, I: t_{1} \mid i, r: t_{0}\right)
\end{aligned}
$$

where $c^{(1)}\left(n, I: t_{1} \mid i, r: t_{0}\right)$ immediately follows from (17), with the appropriate substitutions; the result is

$$
\begin{aligned}
& c^{(1)}\left(n, I: t \mid i, r: t_{0}\right) \\
&=-\left(\frac{2 \pi}{\hbar V}\right)^{1 / 2}\left(\boldsymbol{\mu}^{I i} \cdot \boldsymbol{e}\right)\langle n \mid r\rangle \exp \left[-\mathrm{i}\left(\omega_{I i}-\omega_{0}\right) t_{0}\right] \\
& \times \exp \left[-\mathrm{i}\left(\omega_{0} t_{m}+\phi_{0}\right)\right] \int_{t_{0}}^{t_{1}} J\left(t_{2}\right) \exp \left[\mathrm{i}\left(\omega_{I i}-\omega_{0}\right) t_{2}\right] \mathrm{d} t_{2} .
\end{aligned}
$$

The time integration in (28) can be carried out by making use of the Fourier series for $J\left(t_{2}\right)$, as given by equation (15). Provided $\Delta \omega \ll\left|\omega_{I i}-\omega_{0}\right|$, i.e. provided the bandwidth of the laser radiation is much smaller than the detuning from any resonance, then we have

$$
\begin{aligned}
\int_{t_{0}}^{t_{1}} J\left(t_{2}\right) \exp \left[\mathrm{i}\left(\omega_{I i}-\omega_{0}\right) t_{2}\right] \mathrm{d} t_{2} \\
\simeq \frac{-\mathrm{i}}{\omega_{I i}-\omega_{0}}\left\{J\left(t_{1}\right) \exp \left[\mathrm{i}\left(\omega_{I i}-\omega_{0}\right) t_{1}\right]-J\left(t_{0}\right) \exp \left[\mathrm{i}\left(\omega_{I i}-\omega_{0}\right) t_{0}\right]\right\}
\end{aligned}
$$

and the second term on the right can be ignored in view of the requirement for smooth switching (see equation (21)).

We can now evaluate $c^{(2)}\left(f, k: t \mid i, r: t_{0}\right)$ as given by (27), using the above result in equation (28). The atomic integrals in (27) can be written down immediately, and the field integrals can be calculated after specifying the intermediate states $|n\rangle$. The summations which are to be performed over these states necessitate the use of complete basis sets for each mode, and for this purpose it is convenient to employ a product of number states $\left|n\left(\omega_{l}\right)\right\rangle$ for each frequency component; summations are then taken over $n\left(\omega_{l}\right)$ for each value of $l$, thus enabling closure to be effected. Hence equation (27) gives

$$
\begin{aligned}
& c^{(2)}\left(f, k: t \mid i, r: t_{0}\right) \\
&=-\frac{2 \pi \mathrm{i}}{V} \sum_{I} \frac{\left(\boldsymbol{\mu}^{k I} \cdot \boldsymbol{e}\right)\left(\boldsymbol{\mu}^{I i} \cdot \boldsymbol{e}\right)}{E_{I i}-\hbar \omega_{0}}\langle f \mid r\rangle \exp \left[-\mathrm{i}\left(\omega_{k i}-2 \omega_{0}\right) t_{0}\right] \\
& \times \exp \left[-2 \mathrm{i}\left(\omega_{0} t_{m}+\phi_{0}\right)\right] \int_{t_{0}}^{t} J^{2}\left(t_{1}\right) \exp \left[\mathrm{i}\left(\omega_{k i}-2 \omega_{0}\right) t_{1}\right] \mathrm{d} t_{1}
\end{aligned}
$$

and the ionisation probability at time $t$ is again given by equation (22). The rate of ionisation can now be derived in a manner similar to that demonstrated for singlephoton ionisation in the last section, and the result is as follows:

$$
R(t)=\frac{8 \pi^{3}}{\hbar c^{2}}\left|\sum_{I} \frac{\left(\mu^{k I} \cdot e\right)\left(\mu^{I i} \cdot e\right)}{E_{Y i}-\hbar \omega_{0}}\right|^{2} \rho_{k} I^{2}(t)
$$

with $I(t)$ given by (25). 


\section{Multiphoton ionisation}

We now consider the general case where the combined energy of any $N$ photons in the laser beam suffices to match or exceed the ionisation potential, though the energy of $(N-1)$ photons does not. Hence

$$
(N-1) \hbar \omega_{M}<W \leqslant N \hbar \omega_{-M}
$$

which necessitates the secondary condition

$$
\Delta \omega<\frac{\omega_{0}}{2 N-1} .
$$

Even for relatively high values of $N$, this does not impose any serious restriction on the bandwidth of the laser radiation. The $N$-photon ionisation probability amplitude is again given by equation (8), with the leading contribution coming from the $n=N$ term in (9). Labelling the $(N-1)$ intermediate states with the subscript $i$, so that $\left|I_{i}\right\rangle$ represents an intermediate state of the atom and $\left|n_{i}\right\rangle$ the corresponding state of the field, we have

$$
\begin{aligned}
& c^{(N)}\left(f, k: t \mid i, r: t_{0}\right) \\
&= \frac{\mathrm{i}}{\hbar} \sum_{n_{N-1}} \sum_{I_{N-1}} \int_{t_{0}}^{t} \mathrm{~d} t_{1}\langle k| \exp \left[\mathrm{i} H_{\text {atom }}\left(t_{1}-t_{0}\right) / \hbar\right] \mu \\
& \times \exp \left[-\mathrm{i} H_{\text {: tom }}\left(t_{1}-t_{0}\right) / \hbar\right]\left|I_{N-1}\right\rangle\langle f| \exp \left[\mathrm{i} H_{\text {rad }}\left(t_{1}-t_{0}\right) / \hbar\right] e_{\lambda}^{(+)} \\
& \times \exp \left[-\mathrm{i} H_{\mathrm{rad}}\left(t_{1}-t_{0}\right) / \hbar\right]\left|n_{N-1}\right\rangle c^{(N-1)}\left(n_{N-1}, I_{N-1}: t_{1} \mid i, r: t_{0}\right)
\end{aligned}
$$

hence by using equation (17) for $c^{(1)}\left(f, k: t \mid i, r: t_{0}\right)$, the result for $c^{(N)}\left(f, k: t \mid i, r: t_{0}\right)$ can be found by recursive use of (33). The result is as follows:

$c^{(N)}\left(f, k: t \mid i, r: t_{0}\right)$

$$
\begin{aligned}
= & \left(\frac{2 \pi \hbar}{V}\right)^{N / 2} \frac{\mathrm{i}^{N+1}}{\hbar} \mathscr{2}_{N}\langle f \mid r\rangle \exp \left[-\mathrm{i}\left(\omega_{k i}-N \omega_{0}\right) t_{0}\right] \\
& \times \exp \left[-N \mathrm{i}\left(\omega_{0} t_{m}+\phi_{0}\right)\right] \int_{t_{0}}^{t} J^{N}\left(t_{1}\right) \exp \left[\mathrm{i}\left(\omega_{k i}-N \omega_{0}\right) t_{1}\right] \mathrm{d} t_{1}
\end{aligned}
$$

where

$\mathscr{Q}_{N}=\sum_{I_{1}} \sum_{I_{2}} \ldots \sum_{I_{N-1}} \frac{\boldsymbol{\mu}\left({ }^{k I_{N-1}} \cdot e\right)\left(\boldsymbol{\mu}^{I_{N-1} I_{N-2}} \cdot e\right) \ldots\left(\boldsymbol{\mu}^{I_{1} i} \cdot e\right)}{\left[E_{I_{N-1} i}-(N-1) \hbar \omega_{0}\right]\left[E_{I_{N-2} i}-(N-2) \hbar \omega_{0}\right] \ldots\left(E_{I_{1} i}-\hbar \omega_{0}\right)}$.

In deriving equation (34) it has been assumed that for any intermediate state $\left|I_{P}\right\rangle$, $(1 \leqslant P \leqslant N-1)$, the condition

$$
\Delta \omega \ll\left|\left(\omega_{I_{P}} / P\right)-\omega_{0}\right|
$$

is satisfied: thus the bandwidth of the radiation must be much smaller than the $\omega_{0}$ detuning from any $P$-photon resonance. This is a more severe (though no less reasonable) restriction than that represented by equation (32).

From (34), the rate of $N$-photon ionisation can be derived in the usual way, giving

$$
R(t)=\frac{2 \pi}{\hbar}\left(\frac{2 \pi}{c}\right)^{N}\left|\mathscr{Q}_{N}\right|^{2} \rho_{k} I^{N}(t)
$$


The result has the same form as the conventional Fermi-rule expression, though the latter is strictly valid only in the case of time-invariant irradiance. If the peak intensity is $I_{0}$, so that $I(t)$ can be written as

$$
I(t)=I_{0} G(t)
$$

with $G(t)$ a smooth function taking values between zero and unity, then the ionisation probability can be written in the form

$$
P(t)=\frac{2 \pi}{\hbar}\left(\frac{2 \pi I_{0}}{c}\right)^{N}\left|\mathscr{Q}_{N}\right|^{2} \rho_{k} \tau_{N}
$$

where $\tau_{N}$ is the effective interaction time (Chin and Isenor 1970, Agostini et al 1970), here defined by

$$
\tau_{N}=\int_{t_{0}}^{t} G^{N}\left(t^{\prime}\right) \mathrm{d} t^{\prime} .
$$

\section{Discussion}

In this paper, quantum field theory has been used to describe the pulsed output of a mode-locked laser. By considering a simple atomic photodetection process, it has been shown how the repeated growth and decay of the pulse train can be modelled by use of a coherent-state representation for each mode of the radiation field. Similar methods have then been adopted in deriving expressions for the rate of multiphoton ionisation produced by such mode-locked pulses of light, the results being expressed in terms of the time-dependent irradiance $I(t)$ measured by the photodetector.

The theory can readily be applied to radiation from other kinds of pulsed source, such as giant-pulse lasers. As far as the description of the laser output is concerned, it is shown in the appendix that this may be viewed as the limiting case where the emission spectrum is a narrow but continuous band of frequencies about the mean frequency $\omega_{0}$. The frequency spacing $\Omega$ thus tends to zero, and the pulse repetition time correspondingly tends to infinity, though the bandwidth $\Delta \omega$ remains finite. The frequency distribution of the radiation is then described by the continuous function $A(\omega)$, which we assume is negligible outside the interval $\left(\omega_{0}-\Delta \omega, \omega_{0}+\Delta \omega\right)$. The field theoretic treatment of the single pulse thus has much in common with the classical wavepacket description (see, for example, Bohm 1951), and the corresponding ionisation rates are once again given by the equations in the text, subject to the same specified conditions.

The conventional perturbation-theory treatment of non-resonant ionisation gives an ionisation rate dependent upon the $N$ th power of the irradiance, where $N$ is the number of photons absorbed by each atom. Although this result is strictly applicable only to sources with a time-independent intensity, it has been shown in this paper that the common extension to pulsed sources is justified, as long as the conditions under which the Fermi rule holds remain satisfied. It is worth noting that the switching-on of the interaction has here been handled without the introduction of artificial switching functions, since the laser pulses have a natural growth over a large number of optical cycles. Also, no restrictions have been made on the pulse shape other than the approximation $J\left(t_{0}\right) \simeq 0$, for example in (29). 
The assumption that all frequency components of the laser radiation are far removed from any single-photon or multiphoton resonances raises an interesting point. Although the mean frequency $\omega_{0}$ may be carefully chosen with regard to the atomic system being studied, the restriction still becomes a problem when the bandwidth $\Delta \omega$ of the radiation is large, as in the case of very short pulses. Moreover, the results are applicable only for interaction times $\left(t-t_{0}\right)$ which greatly exceed the optical-cycle length. Since it has already been found possible to produce sub-picosecond pulses of only about 150 cycles duration (Ippen and Shank 1975, Ruddock and Bradley 1976), it seems likely that future technical advances may necessitate the development of a new theory for use in studies involving these pulses.

\section{Acknowledgments}

I acknowledge with thanks many helpful discussions with Professor E A Power and Dr T Thirunamachandran.

\section{Appendix 1. Properties of the temporal envelope function}

The exact form of the temporal envelope function $J(t)$ is determined by the Fourier coefficients in equation (18). The relationship is, however, most clearly demonstrated by rewriting this equation as a Fourier transform, and with this in mind we introduce a smooth function $A(\omega)$, satisfying the condition

$$
A(l \Omega)=a_{l} / \Omega
$$

so that (18) can be expressed as

$$
J(t)=\Omega \sum_{l=-\infty}^{\infty} \int A(\omega) \exp \left[-i \omega\left(t+t_{m}-t_{0}\right) \delta(\omega-l \Omega) \mathrm{d} \omega .\right.
$$

This equation may be more compactly written as

$$
J(t)=\int A(\omega) \operatorname{III}\left(\frac{\omega}{\Omega}\right) \exp \left[-\mathrm{i} \omega\left(t+t_{m}-t_{0}\right)\right] \mathrm{d} \omega
$$

where $\operatorname{III}(x)$ is the shah, or replicating function (Bracewell 1965) defined by

$$
\operatorname{III}(x)=\sum_{n=-\infty}^{\infty} \delta(x-n) .
$$

Using a well known Fourier-transform theorem, the integral (A.1.1) can now be re-expressed as a convolution of the transforms of $A(\omega)$ and $\operatorname{III}(\omega / \Omega)$. If we define

$$
F(t)=\int A(\omega) \exp (-\mathrm{i} \omega t) \mathrm{d} \omega
$$

then since $\operatorname{III}(x)$ is its own Fourier transform we obtain

$$
J(t)=F\left(t+t_{m}-t_{0}\right) * \operatorname{III}\left[\left(t+t_{m}-t_{0}\right) / T\right]
$$

that is

$$
J(t)=\sum_{n=-\infty}^{\infty} F\left(t+t_{m}-t_{0}-n T\right) .
$$


So the envelope function $J(t)$ consists of a superposition of a series of pulses, each with a shape given by the Fourier transform of $A(\omega)$. As a physically realistic example, if $A(\omega)$ fits a Gaussian curve, then $J(t)$ is the superposition of a series of Gaussian pulses (Sargent et al 1974). Since $F(t)$ attains its highest value at the origin, pulses are centred at times $t=t_{0}-t_{m}+n T$; moreover, since $F(t)$ falls off quite rapidly away from the origin, there are troughs between successive pulses where $J(t)$, and hence the irradiance $I(t)$, approaches zero. This accounts for the characteristic train of pulses from the mode-locked laser.

Finally, we note that in the limiting case where the frequency spacing $\Omega \rightarrow 0$, and the periodicity $T \rightarrow \infty$, then obviously only the $n=0$ term contributes to (A.1.4), so from (A.1.3) we find

$$
J(t) \rightarrow \int A(\omega) \exp \left[-\mathrm{i} \omega\left(t+t_{m}-t_{0}\right)\right] \mathrm{d} \omega
$$

in this case $J(t)$ represents a single pulse centred at $t_{0}-t_{m}$.

\section{References}

Agostini P, Barjot G, Mainfray G, Manus C and Thebault J 1970 IEEE J. Quantum Electron. QE-6 782

Andrews D L 1977 J. Phys. B: Atom. Molec. Phys. 10 L659

Bohm D 1951 Quantum Theory (New York: Prentice-Hall)

Bracewell R 1965 The Fourier Transform and its Applications (New York: McGraw-Hill)

Chin S L and Isenor N R 1970 Can. J. Phys. 481445

Crance M and Feneuille S 1977 Phys. Rev. A 161587

Garmire E M and Yariv A 1967 IEEE J. Quantum Electron. QE-3 222

Glauber R J 1963 Phys. Rev. 1312766

Greenhow R C and Schmidt A J 1974 Adv. Quantum Electron. 2158

Gruitter A A, Weber H P and Dändliker R 1969 Phys. Rev. 185629

Ippen E P and Shank C V 1975 Appl. Phys. Lett. 27488

Lompre L A, Mainfray G, Manus C, Repoux S and Thebault J 1976 Phys. Rev. Lett. 36949

Lompre L A, Mainfray G, Manus C and Thebault J 1977 Phys. Rev. A 151604

Loudon R 1973 The Quantum Theory of Light (London: Oxford University Press)

Nussenzveig H M 1973 Introduction to Quantum Optics (London: Gordon and Breach)

Ruddock I S and Bradley D J 1976 Appl. Phys. Lett. 29296

Sargent M, Scully M O and Lamb W E 1974 Laser Physics (Reading, Mass: Addison-Wesley)

Shapiro S L 1977 (ed) Ultrashort Light Pulses: Picosecond Techniques and Applications (Berlin: SpringerVerlag) 